Musées, Patrimoine et Culture scientifiques et techniques

$122 \mid 2009$

mars - avril 2009

\title{
Une galerie d'art pour les sciences
}

Gaël Le Dantec

URL : http://journals.openedition.org/ocim/278

DOI : $10.4000 /$ ocim.278

ISSN : 2108-646X

Éditeur

OCIM

Édition imprimée

Date de publication : 1 mars 2009

Pagination : 5-11

ISSN : 0994-1908

Référence électronique

Gaël Le Dantec, « Une galerie d'art pour les sciences », La Lettre de l'OC/M [En ligne], 122 | 2009, mis en ligne le 01 mars 2011, consulté le 19 avril 2019. URL : http://journals.openedition.org/ocim/278; DOI : $10.4000 /$ ocim. 278 


\section{Les coulisses de la Galerie}

Si la Galerie Industrie \& Recherche a fait peau neuve tout récemment, les contenus sont le fruit d'un travail de toute une équipe depuis plusieurs années déjà. À Cap Sciences, le Pôle Éditions - Actions Industrie \& Recherche réunit six personnes qui entretiennent en permanence un contact avec la communauté scientifique aquitaine et dont le rôle est de produire des outils permettant de présenter au public les innovations et l'actualité des entreprises et des laboratoires. Une équipe qui est renforcée par le détachement d'une ingénieure CNRS, chargée spécifiquement des relations avec les chercheurs.

\section{Les outils}

Les outils de la Galerie sont des films, des visites de sites techniques, des articles d'actualité, des dossiers, des interviews et des portraits de scientifiques...

L'industrie et la recherche sont des secteurs importants en Aquitaine et toutes les actualités de l'innovation ne peuvent être traitées simultanément. Le secret de l'équipe est d'accumuler. Selon l'outil, elle crée chaque semaine, chaque mois, chaque année une nouvelle collection, qui vient compléter les contenus. Les portraits de chercheurs ? Une quarantaine chaque année (depuis 6 ans maintenant, la collection atteint aujourd'hui 240 chercheurs). La version vidéo ? Plus chère : 2 par an seulement soit 15 aujourd'hui. Les articles d'actualité : un par semaine; les interviews de chercheurs : une par mois... Et petit à petit, toujours plus de contacts, de sujets à traiter, de partenaires pour Cap Sciences. Le fichier spécifique du Pôle représente pas moins de 8000 personnes! Comment cela se passe-t-il concrètement ? Premièrement, il faut être en contact permanent avec la communauté scientifique, sortir de Cap Sciences, aller à la rencontre des scientifiques. Et, bien entendu, choisir des outils pertinents, réalisables à un coût abordable et appréciés du grand public.

Prenons pour exemple les portraits vidéo. L'enjeu est de transmettre au public la passion du scientifique pour son métier, son parcours, mais aussi ce qu'il aime, ce qu'il n'aime pas, ce qu'il vit au quotidien...

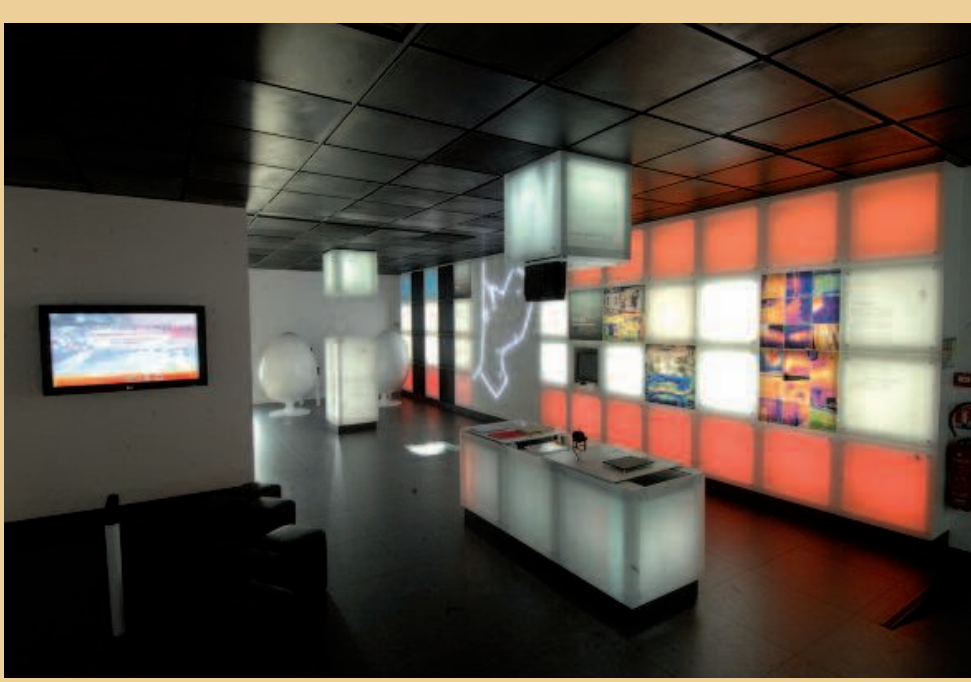

L'espace thématique sur les matériaux propose une présentation de la technologie Infrarouge avec une caméra. (C) Cap Sciences/Frédéric Desmesure

Le tout en trois minutes! On demande au scientifique de nous raconter une histoire. Le format, 3 minutes, a été défini comme un maximum pour tenir en haleine un "visiteur du week-end à Cap Sciences ». Car il ne s'agit pas ici d'un film documentaire où l'on verrait le chercheur dans son environnement, échanger avec des collaborateurs, manipuler sur une paillasse... Ce qui nous intéresse ici c'est la personne en gros plan, et pour rendre l'interview moins statique, deux caméras, qui balayent le personnage sous divers angles et dont l'alternance ou la superposition des images au montage donneront un effet dynamique. Seul artifice auquel le scientifique a droit : un objet qui le représente, celui qu'il veut. Et si une fois un professeur a apporté le dernier livre quiil avait publié... les scientifiques montrent généralement des choses plus personnelles : une boîte à musique, un ballon de rugby, un petit air de guitare, et même un tatouage! Ce concept a été imaginé avec la société bordelaise Burdin Photographies et coûte $1000 € \mathrm{HT}$ par interview. Le scientifique est convié dans un studio d'enregistrement et l'interview est mené par l'un des médiateurs de Cap Sciences pendant environ deux heures. 


\section{Les publics}

La difficulté de la Galerie Industrie \& Recherche est double : elle présente des informations assez techniques (innovations, recherches de pointe...) et s'adresse à des publics très divers : public familial mais aussi professionnels et délégations des collectivités. L'enjeu majeur a donc été de mettre au point des outils et des modes de lecture capables de satisfaire tous les visiteurs. Le public familial et non averti doit avoir envie de pénétrer dans l'espace, pouvoir comprendre facilement ce qui lui est présenté et passer un moment dans un environnement agréable et ludique ; le public de spécialistes et professionnels doit pouvoir utiliser ce lieu comme une vitrine et accueillir des collaborateurs dans un espace à la hauteur et dont les contenus et le niveau d'information sont suffisamment importants.

Contrairement à des expositions plus classiques, le niveau des informations délivrées dans la Galerie Industrie \& Recherche est toujours très technique. En tant que vitrine de l'actualité et des savoir-faire locaux, la Galerie présente des publications récentes dans des domaines tels que les nanotechnologies, les neurosciences, les lasers, des machines nouvellement acquises dans les laboratoires, des produits

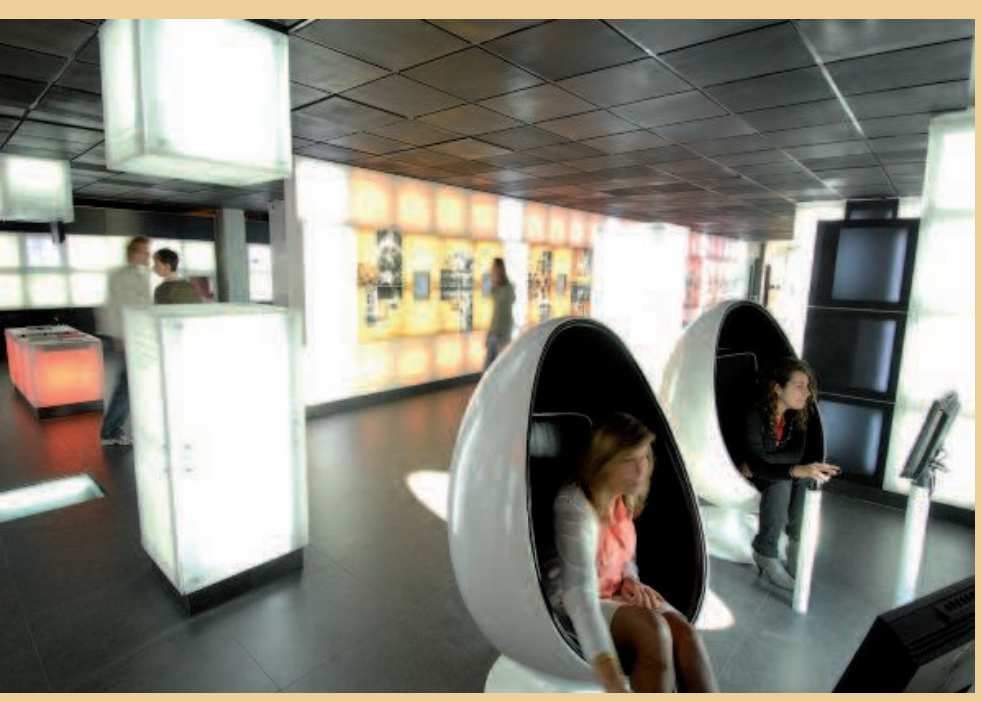

Consultation des bornes actualités (c) Cap Sciences/Frédéric Desmesure de haute technicité. Facilement compréhensibles telles quelles par un public de spécialistes, l'objectif de Cap Sciences est de les rendre lisibles par tous. Comme dans n'importe quelle revue de médiation scientifique, les journalistes du Pôle Actions Industries \& Recherche consacrent autant de temps qu'il faut à étudier, digérer, réécrire et remettre en forme ces informations.

D’un point de vue scénographique, le compromis a été trouvé grâce au design. Ici pas de manips, grands écrans ou objets d'appel traditionnellement utilisés dans les expositions, mais beaucoup de lumière, qui attire l'œil, et un mobilier très accueillant. L'enfant est attiré par les lumières, les recoins, l'adulte peut s'installer confortablement comme dans son salon, et le professionnel, comme dans un espace "VIP », peut y recevoir des collaborateurs. Partout dans la Galerie, des légendes, des signatures, des cartels comme pour des tableaux d'art, viennent donner des chiffres clés, des informations, légender des vitrines ou des images. Jamais un texte ne dépasse une dizaine de lignes. Le style est très épuré, accessible à tous, le texte écrit en noir sur fond blanc.

Pour ceux qui veulent aller plus loin, ce premier niveau de lecture est complété par des bornes multimédia qui présentent des films et des dossiers thématiques. Là encore, un compromis a dû être trouvé et Cap Sciences a fait le pari d'un nouveau type de production vidéo : des films d'animation, d'un format court de moins de 5 minutes permettant de raconter une histoire en voix off, sur un décor animé rassemblant des photographies et des dessins. Pour illustrer le secteur des matériaux, cinq petits films ont ainsi été réalisés en 2007 : les matériaux de la fusée Ariane, le nanotube de carbone, le Pin des Landes, les polymères et les biomatériaux. Ce format a reçu un écho très favorable de la part des visiteurs de Cap Sciences mais la surprise est venue des professionnels et acteurs des collectivités qui, le premier étonnement passé, ont très vite adhéré à ces films originaux, quilis diffusent maintenant dans les salons et les laboratoires.

Marianne Pouget Responsable du Pôle Éditions-Action Industrie \& Recherche à Cap Sciences 


\section{$D^{\prime}$ une vitrine à une galerie}

Depuis sa création, il y a cinq ans, la Galerie Industrie \& Recherche remplissait son rôle de vitrine des capacités scientifiques, techniques et industrielles de l'Aquitaine. Pourtant, les responsables de Cap Sciences voulaient en faire autre chose : un lieu de rencontre et de médiation entre les chercheurs, les industriels dans lequel le public habituel du centre et des visiteurs seraient invité à se faire une idée de la région.

Il aura fallu des années de maturation et des mois de travail, de cogitations, d'essais. On ne passe pas si vite du concept de la vitrine à celui de la galerie et la mue n'est peut-être pas finie.

Au départ l'idée est assez simple : inviter les visiteurs à se laisser surprendre par une autre vision de la recherche et de l'industrie en Aquitaine, le tout dans un lieu unique.

Les pièges sont pourtant nombreux : la vulgarisation, terrain glissant auquel on préfère ici la notion de culture ; l'institutionnel, effet repoussoir garanti ; la petite exposition thématique qui privilégie tel ou tel secteur de la recherche ou de l'industrie régionale ; la plateforme de communication qui transformerait le lieu en maquette publicitaire. Autre difficulté : un espace réduit, $170 \mathrm{~m}^{2}$ seulement pour beaucoup d'informations et un budget d'environ $200000 €$

\section{Une bande-annonce pour la curiosité}

Pour éviter les écueils, Marianne Pouget, responsable du projet, et le trio de direction de Cap Sciences, Bernard Alaux, Bernard Favre et Jean-Alain Pigearias constituent et consultent un comité scientifique, travaillent avec les collectivités et les organismes économiques et professionnels concernés et font appel à leur expérience et à leur imagination.

L'équipe s'appuie d'abord sur les autres activités que le CCSTI organise autour des sciences et de l'industrie régionales : visites d'entreprises, publications, manifestations diverses dont la galerie constitue une sorte de bande annonce qui donne envie de cultiver la curiosité titillée. Dans le même temps, la Galerie devient un lieu de réexploitation des différentes publications et réalisations du pôle industrie et recherche.

Autre atout : les liens tissés avec les laboratoires et les entreprises depuis des années. Un lien de confiance qui permet de bâtir ensemble une image de l'Aquitaine et de son développement économique sans se transformer en agence de communication.
Enfin, les responsables du projet prennent le temps de réfléchir, de ne pas être d'accord, de faire appel à des compétences extérieures : l'architecte du bâtiment, Bernard Schweitzer, qui propose l'aménagement de l'espace, le scénographe, Jean-Loup Walraet et toute l'équipe de décorateurs, qui travaillent sur la déambulation dans l'espace, le responsable des productions, Arnaud Tornier, qui trouve les solutions d'éclairage. Le déclic se fait lorsque les modules de base sont trouvés: des dalles de $60 \mathrm{~cm}$ par $60 \mathrm{~cm}$ rétro éclairées. Au mur, au sol ou en cubes formant des stalagmites ou stalactites, ces dalles interchangeables vont ouvrir des possibilités infinies en maintenant une harmonie de formes, carrées, et de couleurs. Le design global trouvé, il restait à établir le parcours de visite et l'univers des différentes haltes.

\section{La lumière de la Galerie / \\ La Golerie des lumières}

«Dans ses tâtonnements, l'équipe de Cap Sciences a envisagé un moment de pousser le concept de galerie d'art jusqu'à présenter les informations et les objets dans des cadres éclairés comme des tableaux. Finalement, l'utilisation des dalles de plexiglas autorise un éclairage tout aussi original puisque c'est le mobilier qui éclaire la Galerie.

Derrière les plaques translucides, des diodes électroluminescentes éclairent tout l'espace et permettent de colorer cet éclairage à la demande. Tout comme la configuration des dalles, l'éclairage et la couleur sont donc modulables en fonction des transformations de la Galerie ».

Arnaud Tornier

Responsable des productions à Cap Sciences

\section{Un confort culturel}

Dès l'entrée, on foule aux pieds les clichés sur l'Aquitaine, au sens propre puisque sous certaines dalles du sol, des photographies représentent ce que l'on pense connaître de la région : vignes, dunes et autre paysage bucolique de l'arrière pays. C'est à hauteur d'yeux que se trouve le vrai sujet : un panorama en neuf tableaux composés de photographies et de textes accessibles via des écrans tactiles, pour découvrir les grandes activités de la région : aéronautique, bois, laser, glisse... 


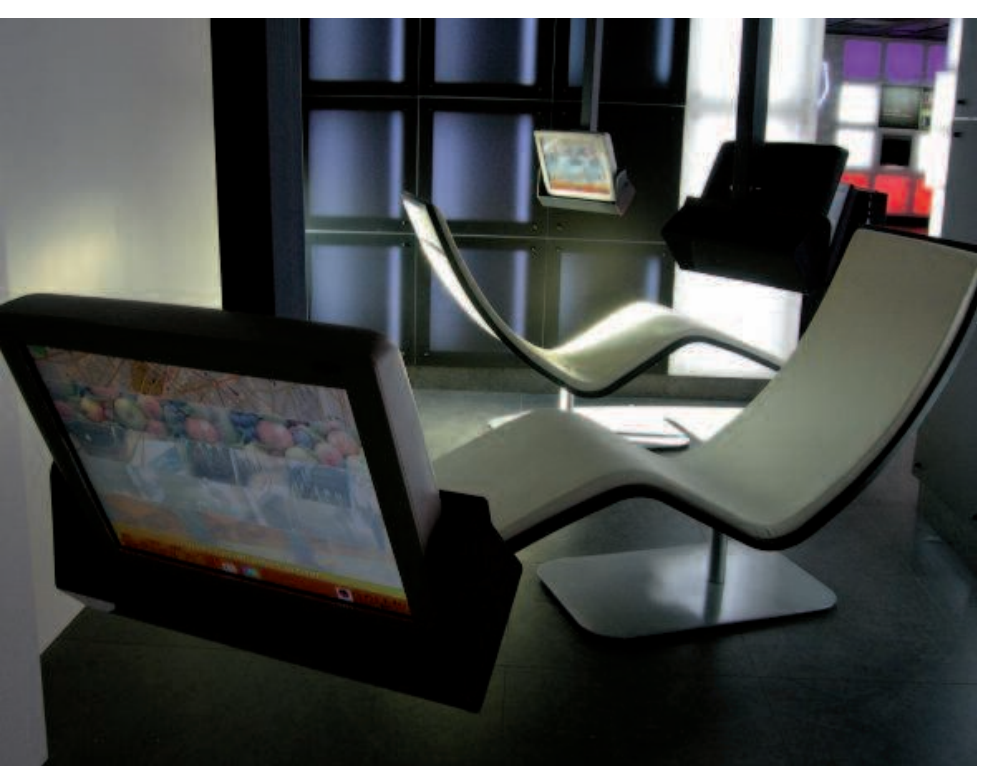

L'espace zoom : des chaises longues pour une consultation individuelle de films sur les entreprises et laboratoires. (c) Cap Sciences/Patrice Brossard

Même si cette entrée en matière est assez réussie esthétiquement et que les textes sont accessibles, on est très vite attiré par ces chaises longues futuristes qui constituent une première occasion, déjà, d'expérimenter ce concept de confort culturel (ou de culture confortable...), c'est-à-dire d'allier le plaisir des sens à celui de la découverte et de la compréhension du monde. On hésite un peu avant de s'allonger car la posture est un piège : il faut s'installer, impossible de se relever et de changer d'activité comme on glisserait discrètement d'un tableau à un autre. Une fois étendu sur un de ces fauteuils genre dentiste, il va falloir se laisser faire. En l'occurrence point de roulette et de néon mais un écran tactile qui permet de choisir parmi une vingtaine de films de dix minutes qui approfondissent des sujets assez variés comme le CHU, le laser Mégajoule ou les énergies en Aquitaine.

Quelques mètres plus loin, la halte suivante est consacrée à la rencontre. "La meilleure façon de rencontrer quelqu'un, explique Bernard Favre, c'est de l'inviter dans son salon ». Ce salon-là est fait pour faire envie : un immense canapé rouge fait face à un très grand écran, placé quasiment au sol. Là encore, on marque une petite hésitation : il y a quelque chose de tout à fait incongru et déstabilisant à se sentir chez soi au milieu d'une galerie mi-art misciences. Les invités sont sur l'écran, juste en face, et une souris en forme de télécommande permet de choisir celui que l'on invite à raconter son histoire et sa passion. Tout est fait pour établir une proximité avec les scientifiques dont on peut également emporter le portrait édité sur des marque-pages.
L'usage dira si cet espace collectif, le canapé qui peut accueillir plusieurs personnes et se trouve au milieu de la Galerie, est compatible avec une rencontre et un esprit qui se veulent intimistes.

L'environnement high-tech, jusque-là réchauffé par le luxe et l'intimité, se refroidit un peu en privilégiant le blanc pour sa partie «Atlas » : une grande carte stylisée de l'Aquitaine rappelle qu'il n'y a pas que Bordeaux et donne quelques chiffres clés. On y découvre que si l'Aquitaine était un village de 100 habitants, 39 travailleraient, 10 seraient à l'école et 3 pratiqueraient le football...

Les visiteurs qui auraient été déstabilisés par l'expérience publique du divan rouge se sentiront tout à fait rassurés par le dispositif suivant. Pour suivre l'actualité de la science et de l'industrie aquitaines, trois sièges en forme de coquille d'œuf invitent à oublier le temps qui passe, grâce à un isolement propice à l'écoute d'émissions de radios réalisées par Cap Sciences ou à la consultation sur écran des dossiers de la revue $H 20$.

\section{Des matériaux à toucher}

Le fond de la galerie en forme de $U$ est consacré à l'exposition thématique qui change chaque année. À l'honneur en 2008 : les matériaux. L'occasion de faire se rencontrer des universitaires et des industriels en leurs proposant un espace pour exposer des objets dans des vitrines, accompagnés de textes rédigés par Cap Sciences.

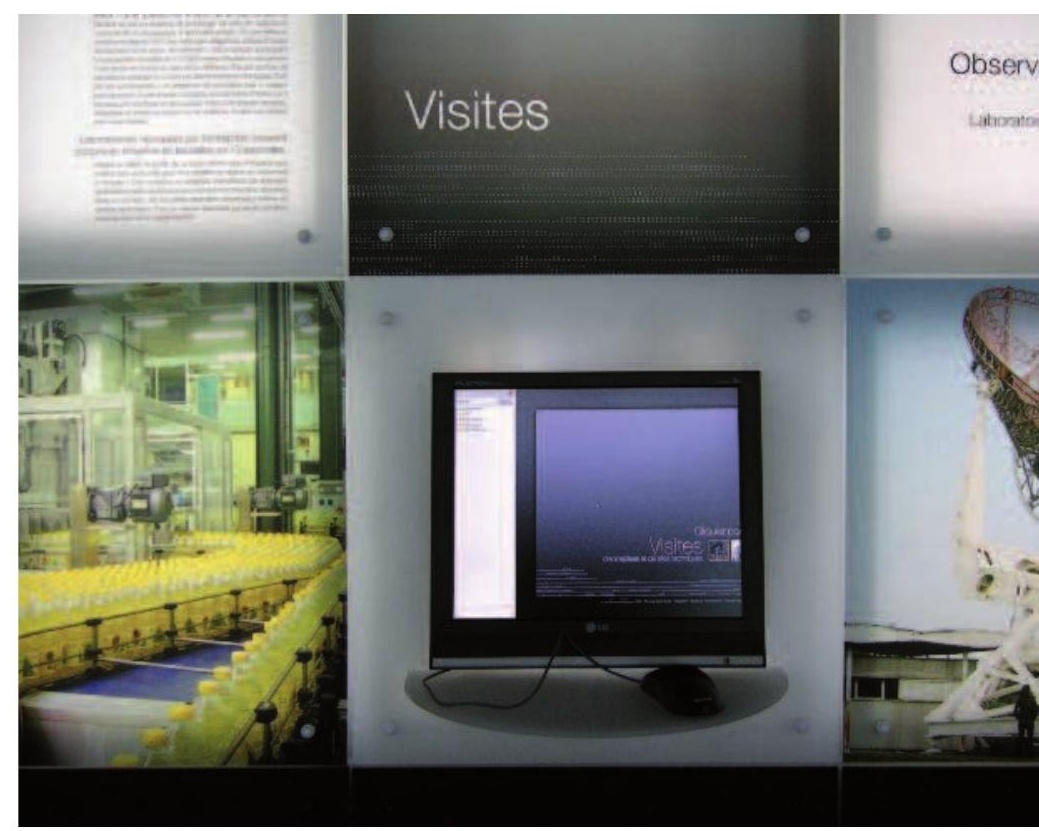

Sur cette borne, le visiteur peut découvrir la liste des 50 sites scientifiques et techniques que Cap Sciences ouvre à la visite. (C) Cap Sciences/Patrice Brossard 


\section{"Ce lieu est un posseur 》}

Interview de Bernard Alaux, directeur de Cap Sciences

Quel est la genèse de la Galerie Industrie \& Recherche ? On se doit d'intégrer l'industrie qui peut être abordée et regardée avec un regard culturel. Les États généraux de la Recherche ont donné comme mission la culture scientifique aux CCSTI. Or on oublie souvent le « $i$ » d'industrie. Pourtant, elle fait bien partie du paysage, des réalités d'un territoire avec une histoire, un contexte, des métiers, de l'innovation... Il est important que le public appréhende cette réalité là et en mesure l'impact sur notre vie de tous les jours.

\section{Vous auriez pu donner cette dimension dans les grandes expositions ?}

Oui, mais là on l'isole et on la traite de façon spécifique. Et dans certaines expositions, il est difficile d'introduire cette dimension là. En tout cas, alors qu'il y a une demande pour appréhender la réalité de ces milieux, les industriels ont des difficultés à permettre au public de venir découvrir leur quotidien, leur univers, leurs métiers. Alors, on a décidé de leur dédier un espace de 200 mètres carrés pour offrir le panorama de la réalité industrielle de ce territoire, y présenter des univers, des métiers, des hommes, des recherches, des transferts.

\section{Il y avait une attente que vous avez pu mesurer ?}

Oui bien sûr. La demande est double. Ce lieu est un passeur et un intermédiaire. Cette demande existe de la part du public et on la vérifie tous les jours quand on fait des visites de sites industriels où on enregistre cinquante personnes par semaine. Cela n'est pas neutre. Et, en amont, l'industrie (la source) souhaite présenter la réalité de son

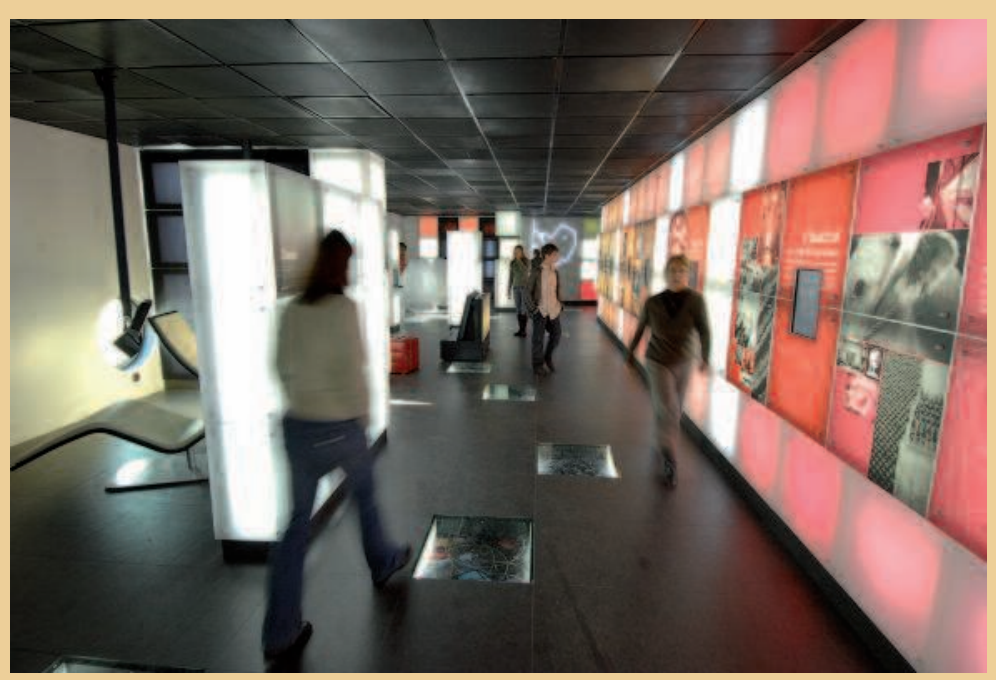

L'entrée de la Galerie et le panorama des filières d'activités en Aquitaine (c) Cap Sciences/Frédéric Desmesure secteur. Il faut faire la différence entre communication, information et culture. On ne doit pas transformer la Galerie en espace de communication et de mise en valeur mais en un lieu où l'on fait appréhender la dimension culturelle (le contexte, l'impact, l'évolution) pour mieux comprendre pourquoi en Aquitaine, tel ou tel secteur industriel est présent.

\section{Quel est le rôle des collectivités territoriales ?}

Les collectivités qui accompagnent le développement industriel et économique ont besoin d'expliquer au public les raisons de leurs choix. La Galerie concourt et participe à présenter en un coup d'œil une région et un territoire. Et comme nos outils sont transportables, cela permet de présenter l'industrie en Aquitaine en dehors de Cap Sciences, lors de salons ou dans d'autres lieux.

\section{Pourquoi un espace permanent comme la Galerie ?}

Toute personne qui pénètre dans une région, le fait par l'aéroport, l'autoroute, l'Internet mais dans aucun de ces endroits il y a une sorte de kiosque d'information de la réalité $d u$ territoire. On a donc pensé un espace de présentation pour le grand public, pour qu'il ait une vue d'ensemble.

Cet espace permet de susciter des débats par des croisements de regards sur des thèmes différents. On peut imaginer ici quels seraient les transferts de technologie possibles, des idées de développement... En ayant un espace permanent, on peut générer ce type de rencontres que l'on doit développer à Cap Sciences.

On matérialise les choses, on leur enlève leur côté abstrait. On visualise mieux. On peut toucher les objets, ce n'est pas la même chose que lire une encyclopédie ou une publication institutionnelle.

La Galerie est aussi devenue un acteur qui permet d'impliquer des associations professionnelles pour développer le versant culturel de leur secteur. Elles n'ont pas beaucoup de présentation de leur propre filière (histoire, prospective, innovation, métiers...). En mettant les outils que l'on crée à leur disposition, on optimise les moyens.

\section{Est-ce que les industriels comprennent bien ce rôle de} la Galerie?

La Galerie n'est pas encore assez utilisée par eux. Ils commencent à comprendre l'intérêt du lieu, à vouloir y faire des évènements. Prochainement le directeur d'un laboratoire amène toute son équipe visiter la Galerie. Il veut faire découvrir toutes les entreprises et laboratoires de la filière que ses employés ne connaissent peut-être même pas. Il les emmène ici pour leur offrir un panorama de ce qui se fait et leur ouvrir un peu l'esprit. Pour l'avenir, il faut que l'on favorise ce genre de lien. 
Lidée est de pousser un peu plus loin le concept de galerie d'art en montrant des objets sans faire de pédagogie autour. C'est l'occasion de signifier la valeur et pourquoi pas la beauté d'un matériau plutôt que d'un produit complexe.

Les objets entiers, palpables, peuvent être présentés au cœur de l'exposition dans un espace dédié à des animations. Enfin des films sont également présentés, réalisés par ou avec Cap Sciences. Simple sans être simpliste, celui sur les nanotubes de carbones, par exemple, raconte le processus de l'innovation tout en laissant entrevoir les applications potentielles grâce à un mélange très vivant d'images vidéo et d'animations.

Un effet de perspectives donne au couloir qui mène doucement vers la sortie une impression de longueur étonnante. Consacrés à un quiz, à un aperçu des visites d'entreprises organisées par Cap Sciences et à des portraits de recherches régionales, textes, photographies et bornes informatiques se succèdent en douceur, unifiés par les cubes interchangeables qui les abritent, tandis que le mur d'en face prolonge l'effet galerie d'art en proposant un reportage photographique.
On ressort de cette galerie en ayant oublié que l'on se trouvait dans le hangar de Cap Sciences tant le design si particulier y crée une atmosphère originale.

L'équipe de Cap Sciences promet d'enrichir encore le lieu en faisant appel à des artistes pour qu'ils achèvent de transformer la vitrine en galerie en donnant leurs visions des grands secteurs du développement économique régional. Ajouté au fait que les modules qui constituent la structure de la Galerie sont modifiables à volonté et que les données multimédias s'enrichissent constamment, il est possible que le public revienne régulièrement, en tout cas celui qui aura été séduit. 